\title{
TWO-STEP CAMERA CALIBRATION METHOD DEVELOPED FOR MICRO UAV'S
}

\author{
M. Gašparović, $\mathrm{PhD}^{\mathrm{a}, *}$, Assist. Prof. Dubravko Gajski, $\mathrm{PhD}^{\mathrm{b}}$ \\ ${ }^{a}$ GEOF, Faculty of Geodesy, University of Zagreb, Kačićeva 26, Zagreb, Croatia - mgasparovic@geof.hr \\ ${ }^{\mathrm{b}}$ GEOF, Faculty of Geodesy, University of Zagreb, Kačićeva 26, Zagreb, Croatia - dgajski@geof.hr
}

Commission I, ICWG I/Vb

KEY WORDS: Photogrammetry, two-step camera calibration, micro UAV, ultra wide lenses

\begin{abstract}
:
The development of unmanned aerial vehicles (UAVs) and continuous price reduction of unmanned systems attracted us to this research. Professional measuring systems are dozens of times more expensive and often heavier than "amateur", non-metric UAVs. For this reason, we tested the DJI Phantom 2 Vision Plus UAV. Phantom's smaller mass and velocity can develop less kinetic energy in relation to the professional measurement platforms, which makes it potentially less dangerous for use in populated areas. In this research, we wanted to investigate the ability of such non-metric UAV and find the procedures under which this kind of UAV may be used for the photogrammetric survey. It is important to emphasize that UAV is equipped with an ultra wide-angle camera with 14MP sensor. Calibration of such cameras is a complex process. In the research, a new two-step process is presented and developed, and the results are compared with standard one-step camera calibration procedure. Two-step process involves initially removed distortion on all images, and then uses these images in the phototriangulation with self-calibration. The paper presents statistical indicators which proved that the proposed two-step process is better and more accurate procedure for calibrating those types of cameras than standard one-step calibration. Also, we suggest two-step calibration process as the standard for ultra-wideangle cameras for unmanned aircraft.
\end{abstract}

\section{INTRODUCTION}

The market of UAVs is experiencing a boom in recent years, especially the development of small, easily manageable and mainly autonomous system. Those kinds of UAVs are usually equipped with an autonomous navigation system (GPS + INS) and digital camera. According to the measurement characteristics, those systems can be divided into systems designed for photogrammetric purposes (metric) and systems that are not (non-metric). Although photogrammetric systems generally have better and declared measurement characteristics than non-metric systems, they have some limitations, which strongly reduce the possibility of applying such systems. Metric systems are 10-30 times more expensive than non-metric systems (Zalović 2015). Systems for non-professional (recreational) use are subjected to far more lenient terms and conditions of flying and due to less mass and velocity, can produce less kinetic energy, which makes them potentially less dangerous for use in populated areas (CCAA 2015). For a nonmetric system to be used for measuring purposes, it is necessary to calibrate the digital camera. Many scientists (Perez et al. 2013) have researched analytical calibration procedures. High values of distortion in non-metric digital cameras have a very negative influence on the accuracy and reliability of the determination of calibration elements (Karara 1989). For this reason, this paper suggests that most of the image distortion should be removed in the first step and then the final calibration should be carried out.

\section{DATA ACQUISITION}

Today, unmanned aircrafts are increasingly used for the photogrammetric survey of small areas or high buildings. Those aircrafts can be divided into several categories, which are shown in Table 1. Legal regulations specify and allow the use of certain aircrafts for the purpose of the aerial survey. In this paper, we dealt with micro UAVs because of their wide availability and lower legal restrictions. Of course, those kinds of aircrafts due to the load usually have a non-metric camera, often with a super wide-angle lens. It was also the reason for the development of a two-step calibration for lenses with large radial and tangential distortion values.

Table 1. Classification of UAVs according to Unmanned Aerial Vehicle Systems Association (Bendea et al. 2008)

\begin{tabular}{|c|c|c|c|c|c|}
\hline 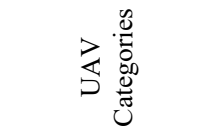 & 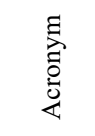 & $\begin{array}{l}\stackrel{8}{\infty} \\
\stackrel{\Xi}{\approx} \\
\approx\end{array}$ & $\underset{\stackrel{\Xi}{\Xi}}{\stackrel{\Xi}{\Xi}}$ & 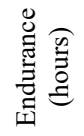 & 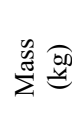 \\
\hline Micro & $\mu$ & $<10$ & 250 & 1 & $<5$ \\
\hline Mini & Mini & $<10$ & $\begin{array}{l}150- \\
300\end{array}$ & $<2$ & 150 \\
\hline Close Range & $\mathrm{CR}$ & $10-30$ & 3000 & $2-4$ & 150 \\
\hline Short Range & SR & $30-70$ & 3000 & $3-6$ & 200 \\
\hline Medium Range & MR & $70-200$ & 5000 & $6-10$ & 1250 \\
\hline $\begin{array}{l}\text { Medium Range } \\
\text { Endurance }\end{array}$ & MRE & $>500$ & 8000 & $\begin{array}{l}10- \\
18 \\
\end{array}$ & 1250 \\
\hline $\begin{array}{c}\text { Low Altitude } \\
\text { Deep Penetration }\end{array}$ & LADP & $>250$ & $\begin{array}{c}50- \\
9000 \\
\end{array}$ & $0,5-1$ & 350 \\
\hline $\begin{array}{c}\text { Low Altitude } \\
\text { Long Endurance }\end{array}$ & LALE & $>500$ & 3000 & $>24$ & $<30$ \\
\hline $\begin{array}{l}\text { Medium Altitude } \\
\text { Long Endurance }\end{array}$ & MALE & $>500$ & 14000 & $\begin{array}{c}24- \\
48 \\
\end{array}$ & 1500 \\
\hline
\end{tabular}

\footnotetext{
* Corresponding author
} 
Micro UAV DJI Phantom 2 Vision Plus (Figure 1) was used for this study. Aircraft has a built-in digital camera with the focal length of $5 \mathrm{~mm}$, and the dimension of the sensor $4384 \times 3288 \mathrm{px}$ (14MP). The camera field of view across the width of the sensor is $110^{\circ}$ and $85^{\circ}$ across the height of the sensor.

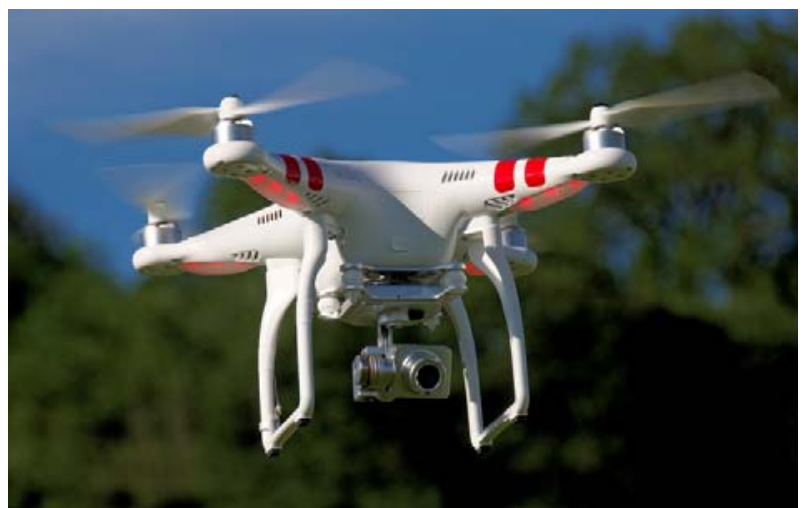

Figure 1. Micro UAV DJI Phantom 2 Vision Plus (URL 1)

A test field of 105 evenly distributed points was used for testing and the calibration of images (Figure 2). Coordinates of the test field were determined by the space intersections taken from more occupation-points measured by the total station Leica TCR705. The achieved accuracy of the coordinates of the test field is $\pm 0.1 \mathrm{~mm}$.

$$
\begin{aligned}
& 9192939499596979899 \quad 100101102103104105
\end{aligned}
$$

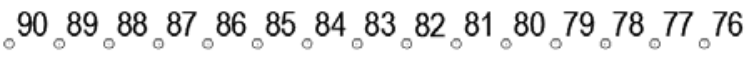

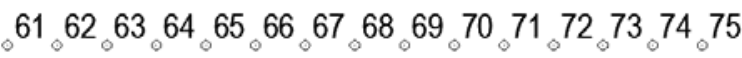

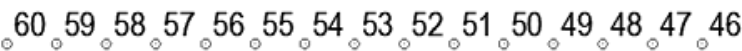

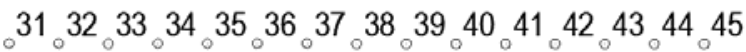

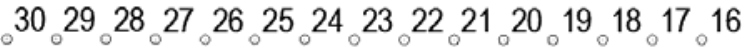

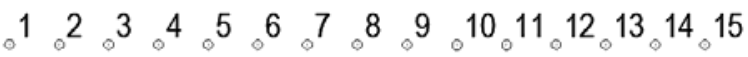

After completing the test field survey with the UAV, images that are used in the research were selected. The images were selected to cover different perspectives and scales of the test fields.

\section{SPATIAL CALIBRATION}

Mathematical model of central projection, which describes the ideal central projection of the object coordinates (in three dimensions) to the image plane (in two dimensions) describe collinearity equations (1) (Kraus 2006):

$$
\begin{aligned}
& x=x_{0}-c \frac{r_{11}\left(X-X_{0}\right)+r_{21}\left(Y-Y_{0}\right)+r_{31}\left(Z-Z_{0}\right)}{r_{13}\left(X-X_{0}\right)+r_{23}\left(Y-Y_{0}\right)+r_{33}\left(Z-Z_{0}\right)} \\
& y=y_{0}-c \frac{r_{12}\left(X-X_{0}\right)+r_{22}\left(Y-Y_{0}\right)+r_{32}\left(Z-Z_{0}\right)}{r_{13}\left(X-X_{0}\right)+r_{23}\left(Y-Y_{0}\right)+r_{33}\left(Z-Z_{0}\right)},
\end{aligned}
$$

where are:

$x, y$ - Image coordinates of the measured point $T$

$x_{0}, y_{0}$ - Image coordinates of the principal point

$c$ - Calibrated focal length

$r_{i j}$ - Elements of rotation matrix

$X, Y, Z$ - Ground coordinates of point $T$

$X_{0}, Y_{0}, Z_{0}$ - Ground coordinates of projection center.

Real projection differs from the above-mentioned ideal case due to imperfections in the development of the lens. Mapping error formed thereby is called lens distortion. Lens distortion is an error of projection that does not affect the sharpness only the geometric deformation of the image compared to an ideal central projection (McGlone 2013).

Total corrections for the elimination of distortion in both directions of the image coordinate system are obtained by summing the individual variables (equation 2). Those variables describe the partial systemic effects, whose geometric meaning is given in Table 2. The influence of each variable to the total correction is determined by coefficient $a_{i}$, which is determined by the method of spatial calibration (Kager et al. 2002):

$$
\begin{aligned}
& d X=\sum_{i \in \hat{l}}\left[a_{i} \cdot d X_{i}(x, y)\right] \\
& d Y=\sum_{i \in \hat{l}}\left[a_{i} \cdot d Y_{i}(x, y)\right] .
\end{aligned}
$$

Corrected image coordinates $(X, Y)$ for each measuring point on image $\left(X^{\prime}, Y^{\prime}\right)$ are calculated by the following equations (Kager et al. 2002):

$$
\begin{aligned}
& X=X^{\prime}+d X(x, y) \\
& Y=Y^{\prime}+d Y(x, y),
\end{aligned}
$$

where $x$ and $y$ are normalized coordinates (Kager et al. 2002):

$$
\begin{aligned}
& x=\left(X^{\prime}-X_{0}\right) / \rho_{0} \\
& y=\left(Y^{\prime}-Y_{0}\right) / \rho_{0} \\
& r^{2}=x^{2}+y^{2},
\end{aligned}
$$

where are:

$X_{0}, Y_{0}$ - Image coordinates of the principal point $\rho_{0}$ - Normalization radius.

Parameters which are recommended by the Institute of Photogrammetry and Remote Sensing at the Technical University of Vienna were selected for modeling of systematic distortion. The mathematical model and geometrical interpretation of each of those parameters are given in Table 2.

Table 2. Distortion variables according to TU-standard (Kager et al. 2002)

\begin{tabular}{|l|l|l|l|}
\hline$i \in \hat{l}$ & $d X_{i}(x, y)$ & $d Y_{i}(x, y)$ & Geometric meaning \\
\hline 1 & 0 & $x$ & Affinity - skewness of axes \\
\hline 2 & 0 & $y$ & Affinity - scaling of y-axis \\
\hline 3 & $x \cdot\left(r^{2}-1\right)$ & $y \cdot\left(r^{2}-1\right)$ & Radial distortion, 3. degree \\
\hline 4 & $x \cdot\left(r^{4}-1\right)$ & $y \cdot\left(r^{4}-1\right)$ & Radial distortion, 5. degree \\
\hline 5 & $r^{2}+2 \cdot x^{2}$ & $2 \cdot x \cdot y$ & Tangential distortion \\
\hline 6 & $2 \cdot x \cdot y$ & $r^{2}+2 \cdot y^{2}$ & Tangential distortion \\
\hline 37 & $x \cdot\left(r^{6}-1\right)$ & $y \cdot\left(r^{2}-1\right)$ & Radial distortion, 7. degree \\
\hline
\end{tabular}

Previously described mathematical model was used in phototriangulation with self-calibration on the original images 
and on the images where distortion was previously removed. Phototriangulation was calculated with BBA method (Bundle Block Adjustment) by using ORPHEUS 3.2.1 software developed at the Technical University of Vienna. All processes in this research are shown in Figure 3.

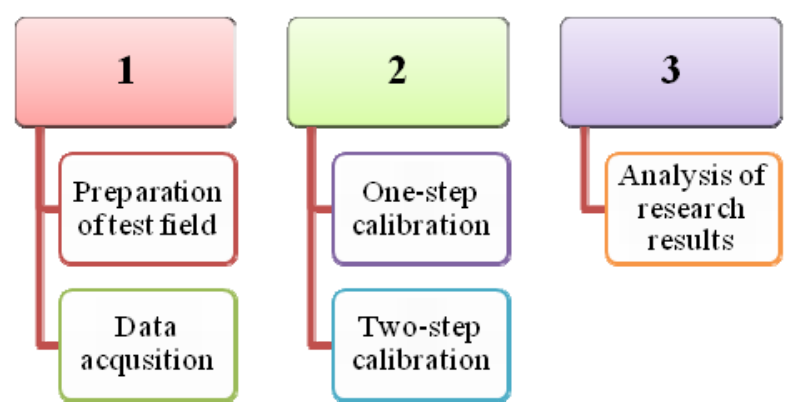

Figure 3. Steps of the research

\subsection{One-step calibration}

This chapter explains the procedure of phototriangulation with self-calibration on the original images. This procedure can be called a one-step calibration or standard calibration procedure.

\subsubsection{Initial value}

Initial elements of interior camera orientation are calculated using the following equations:

$$
\begin{aligned}
& x_{0}=\frac{w_{\mathrm{px}}}{2}=2192 \mathrm{px} \\
& y_{0}=\frac{h_{\mathrm{px}}}{2}=1644 \mathrm{px} \\
& w_{\mathrm{mm}}=c_{m m} \cdot \arctan \left(110^{\circ}\right)=5,452 \mathrm{~mm} \\
& h_{\mathrm{mm}}=c_{m m} \cdot \arctan \left(85^{\circ}\right)=4,887 \mathrm{~mm} \\
& c=\frac{1}{2}\left(\frac{w_{\mathrm{px}}}{w_{\mathrm{mm}}}+\frac{h_{\mathrm{px}}}{h_{\mathrm{mm}}}\right) \cdot c_{\mathrm{mm}}=3663 \mathrm{px},
\end{aligned}
$$

where $w_{\mathrm{px}}$ and $h_{\mathrm{px}}$ are width and height of the image matrix (4384 px, $3288 \mathrm{px}$ ), $w_{\mathrm{mm}}$ and $h_{\mathrm{mm}}$ calculated sensor width and height, $x_{0}$ and $y_{0}$ position of principal point, $c_{\mathrm{mm}}$ focal length $(5 \mathrm{~mm}), c$ focal length in pixels and value $110^{\circ}$ and $85^{\circ}$ representing camera field of view (FOV) in direction of the height or width of the sensor. The values shown above are taken from camera and lens specifications. Manufacturer does not provide sensor dimension in $\mathrm{mm}$, therefore, this information was calculated from camera FOV.

Phototriangulation is a simultaneous process of finding the parameters of spatial reconstruction of bundles implemented by the least squares method with the assessment of accuracy (Triggs et al. 2000). In this adjustment unknowns are all parameters of interior orientation: coordinates of the principal point, focal length and distortion parameters shown in Table 2. Normalization radius was determined by previous research so the curve of the radial distortion is balanced. Thus, the normalization radius was calculated by the following equation based on the research Gajski and Gašparović 2015:

$$
\begin{aligned}
& d_{\text {D90 }}=\sqrt{2144^{2} \cdot 1424^{2}}=2574 \mathrm{px} \\
& d_{\text {PHANTOM }}=\sqrt{2192^{2} \cdot 1644^{2}}=2740 \mathrm{px} \\
& \rho_{0}=\rho_{0}^{D 90} \cdot \frac{d_{\text {PHANTOM }}}{d_{D 90}} \approx 2000 \mathrm{px}
\end{aligned}
$$

where $d_{\mathrm{D} 90}$ and $d_{\text {PHANTOM }}$ are semi diagonals of image matrix obtained by Nikon D90 camera (Gajski and Gašparović 2015) and UAV Phantom camera, $\rho_{0}$ normalization radius of Phantom camera and $\rho_{0}^{D 90}$ normalization radius of Nikon D90 camera.

\subsubsection{Self-calibration}

Self-calibration is the process of conducting phototriangulation where elements of interior orientation (focal length $\mathrm{c}$, the position of the principal point $x_{0}, y_{0}$ and distortion elements of camera lens) are introduced as unknowns into the adjustment. Unknown in the adjustment are also the coordinates of GCP (Ground Control Points) as well as the elements of the exterior orientation. Numerous authors have researched the selfcalibration process: Armstrong 1996, Pollefeys 1999 and Azizi 2003.

The reference standard deviation of phototriangulation with self-calibration on original images is:

$$
\mathrm{s}_{0}=1,3326 \mathrm{px} \text {. }
$$

Adjusted values of the exterior orientation parameters for images 540, 542 and 561 (Tables 3 and 4) are attached in the tables below. These images contain the biggest amount of the ground control points (GCPs) from all images in adjustment. One of the most important statistical indicators in adjustment is the trace of covariance-matrix of unknowns.

Table 3. The angular exterior parameters of images and standard deviation

\begin{tabular}{|c|c|c|c|c|c|c|}
\hline IMG & $\begin{array}{c}\omega \\
{[\mathrm{grad}]}\end{array}$ & $\begin{array}{c}s_{\omega} \\
{[\mathrm{grad}]}\end{array}$ & $\begin{array}{c}\varphi \\
{[\mathrm{grad}]}\end{array}$ & $\begin{array}{c}s_{\varphi} \\
{[\mathrm{grad}]}\end{array}$ & $\begin{array}{c}\kappa \\
{[\mathrm{grad}]}\end{array}$ & $\begin{array}{c}S_{\kappa} \\
{[\mathrm{grad}]}\end{array}$ \\
\hline 540 & -83.63850 & 0.0492 & 131.24567 & 0.0412 & 101.45593 & 0.0283 \\
\hline 542 & -97.09087 & 0.0465 & 128.31638 & 0.0395 & 104.71013 & 0.0274 \\
\hline 561 & -98.63338 & 0.0368 & 100.34454 & 0.0347 & 101.13206 & 0.0138 \\
\hline trace & \multicolumn{6}{|c|}{$4.367 \mathrm{E}-06(\mathrm{sd}=0.0362)$} \\
\hline
\end{tabular}

Table 4. Coordinates of image projection centers and standard deviation

\begin{tabular}{|c|c|c|c|c|c|c|}
\hline IMG & $\begin{array}{c}X \\
{[\mathrm{~m}]}\end{array}$ & $\begin{array}{c}S_{X} \\
{[\mathrm{~m}]}\end{array}$ & $\begin{array}{c}Y \\
{[\mathrm{~m}]}\end{array}$ & $\begin{array}{c}S_{Y} \\
{[\mathrm{~m}]}\end{array}$ & $\begin{array}{c}Z \\
{[\mathrm{~m}]}\end{array}$ & $\begin{array}{c}S_{Z} \\
{[\mathrm{~m}]}\end{array}$ \\
\hline 540 & 108.231 & 0.0040 & 103.218 & 0.0068 & 101.048 & 0.0034 \\
\hline 542 & 105.053 & 0.0030 & 103.194 & 0.0071 & 101.026 & 0.0031 \\
\hline 561 & 104.116 & 0.0022 & 106.299 & 0.0041 & 103.743 & 0.0035 \\
\hline trace & \multicolumn{7}{|c|}{$1.900 \mathrm{E}-03(\mathrm{sd}=0.0032)$} \\
\hline
\end{tabular}

Also, in this adjustment as unknowns are involved and determined interior orientation parameters. Below are shown the values of these parameters, their standard deviations and matrix trace with part of unknowns related to the basic interior orientation parameters trace $_{\text {IOmain }}$ and additional, distorting parameters of interior orientation trace $_{\text {IOdist }}$. 


$$
\begin{aligned}
& x_{0}=2191.57080 \mathrm{px}(\mathrm{sd}=1.4406) \\
& y_{0}=1736.24597 \mathrm{px}(\mathrm{sd}=1.5190) \\
& c=2053.01514 \mathrm{px}(\mathrm{sd}=1.5717) \\
& \text { trace }_{\text {IOmain }}=3.859(\mathrm{sd}=1.5114) \\
& a_{1}=-1.55946(\mathrm{sd}=0.512) \\
& a_{2}=-2.66556(\mathrm{sd}=0.548) \\
& a_{3}=-147.686(\mathrm{sd}=1.95) \\
& a_{4}=123.685(\mathrm{sd}=2.62) \\
& a_{5}=-6.94164(\mathrm{sd}=0.377) \\
& a_{6}=-13.8216(\mathrm{sd}=0.317) \\
& a_{37}=-33.2420(\mathrm{sd}=1.07) \\
& \operatorname{trace}_{\text {IOdist }}=7.123(\mathrm{sd}=1.3442) .
\end{aligned}
$$

\subsection{Two-step calibration}

This chapter explains the process of phototriangulation with self-calibration on images with initially removed distortion (Figure 4). This process can be called two-step calibration. Initial removal of distortion on all images was done according to manufacturer's instructions.

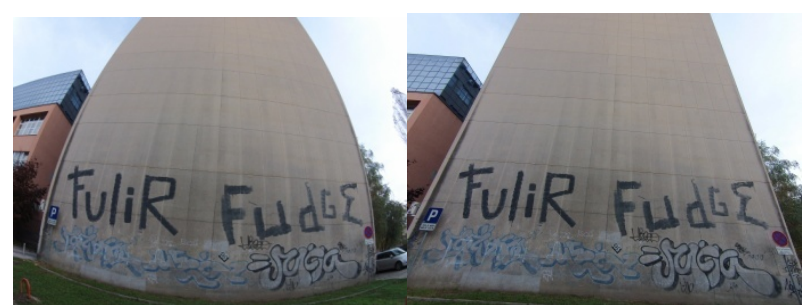

Figure 4. Image 542: left - original, right - with the initially removed distortion

Self-calibration was performed after preprocessing the images. The main task of the self-calibration is to determine interior orientation parameters together with additional parameters for lens distortion (Table 2). The reference standard deviation of phototriangulation with self-calibration on preprocessed images is:

$$
\mathrm{s}_{0}=1.6092 \mathrm{px}
$$

Below are attached adjusted values of the exterior orientation parameters for images 540, 542 and 561 (Tables 5 and 6). These images contain most of GCPs among all images in the adjustment. The trace of the covariance matrix of unknown parameters is regarded as one of the most important statistical indicators in the adjustment.

Table 5. The angular exterior parameters of images and standard deviation

\begin{tabular}{|c|c|c|c|c|c|c|}
\hline IMG & $\begin{array}{c}\omega \\
{[\mathrm{grad}]}\end{array}$ & $\begin{array}{c}S_{\omega} \\
{[\mathrm{grad}]}\end{array}$ & $\begin{array}{c}\varphi \\
{[\mathrm{grad}]}\end{array}$ & $\begin{array}{c}S_{\varphi} \\
{[\mathrm{grad}]}\end{array}$ & $\begin{array}{c}\kappa \\
{[\mathrm{grad}]}\end{array}$ & $\begin{array}{c}S_{\kappa} \\
{[\mathrm{grad}]}\end{array}$ \\
\hline 540 & -83.37917 & 0.0254 & 130.99297 & 0.0199 & 101.61299 & 0.0163 \\
\hline 542 & -96.84641 & 0.0230 & 128.07642 & 0.0189 & 104.83316 & 0.0156 \\
\hline 561 & -98.43562 & 0.0211 & 100.20083 & 0.0228 & 101.15824 & 0.0132 \\
\hline trace & \multicolumn{7}{|c|}{$1.429 \mathrm{E}-06(\mathrm{sd}=0.0204)$} \\
\hline
\end{tabular}

Table 6. Coordinates of image projection centers and standard deviation

\begin{tabular}{|c|c|c|c|c|c|c|}
\hline IMG & $\begin{array}{c}X \\
{[\mathrm{~m}]}\end{array}$ & $\begin{array}{c}s_{X} \\
{[\mathrm{~m}]}\end{array}$ & $\begin{array}{c}Y \\
{[\mathrm{~m}]}\end{array}$ & $\begin{array}{c}S_{Y} \\
{[\mathrm{~m}]}\end{array}$ & $\begin{array}{c}Z \\
{[\mathrm{~m}]}\end{array}$ & $\begin{array}{c}S_{Z} \\
{[\mathrm{~m}]}\end{array}$ \\
\hline 540 & 108.231 & 0.0036 & 103.221 & 0.0051 & 101.041 & 0.0034 \\
\hline 542 & 105.054 & 0.0030 & 103.196 & 0.0053 & 101.014 & 0.0032 \\
\hline 561 & 104.111 & 0.0021 & 106.294 & 0.0036 & 103.728 & 0.0023 \\
\hline trace & \multicolumn{6}{|c|}{$1.286 \mathrm{E}-03(\mathrm{sd}=0.0031)$} \\
\hline
\end{tabular}

Also, in this adjustment as unknowns are involved and determined interior orientation parameters. Below we can see the values of these parameters, their standard deviations and matrix trace with part of unknown parameters related to the basic interior orientation parameters trace $_{\text {IOmain }}$ and additional, distorting parameters of interior orientation trace $_{\text {IOdist }}$.

$$
\begin{aligned}
& x_{0}=2184.30371 \mathrm{px}(\mathrm{sd}=0.4316) \\
& y_{0}=1745.79333 \mathrm{px}(\mathrm{sd}=0.4286) \\
& c=2053.46362 \mathrm{px}(\mathrm{sd}=1.2411) \\
& \operatorname{trace}_{\text {IOmain }}=0.7377(\mathrm{sd}=0.7980) \\
& a_{1}=-0.839583(\mathrm{sd}=0.519) \\
& a_{2}=-0.988194(\mathrm{sd}=0.529) \\
& a_{3}=-142.052(\mathrm{sd}=1.85) \\
& a_{4}=118.204(\mathrm{sd}=2.49) \\
& a_{5}=-9.34810(\mathrm{sd}=0.149) \\
& a_{6}=-15.6698(\mathrm{sd}=0.127) \\
& a_{37}=-31.5231(\mathrm{sd}=1.00) \\
& \operatorname{trace}_{\text {IOdist }}=4.330(\mathrm{sd}=1.2657) .
\end{aligned}
$$

\section{ANALYSIS OF RESEARCH RESULTS}

From the statistical indicators presented in previous chapters it is clear that the standard deviations of the angular elements of exterior orientation on the same images are up to 2 times better in a two-step calibration. Also, the linear elements of exterior orientation have smaller standard deviation in two-step calibration. The values on the main diagonal of covariance matrix show the same trend (Tables 3 and 5 for angular elements and tables 4 and 6 for linear elements).

When analyzing the reliability of the calculated elements for the interior orientation, improvements in the two-step calibration are also visible. Standard deviation for $x_{0}$ and $y_{0}$ is up to 3 times better in the two-step calibration in comparison to the one-step calibration. Distortion parameters, determined by twostep calibration have better reliability.

From previously conducted research and comparative results of Phantom camera calibration it can be said that the two-step process is better, and it is recommended for the specified camera.

\section{CONCLUSIONS}

Experimental studies in this paper clearly show that the micro UAVs can be successfully used in the measurement purposes. Previously, systematic errors that cause a geometric deformation of image content in relation to the mathematical model of an ideal central projection should be removed. The greatest influence on the measurement properties of the image has the distortion of the lens. This effect is particularly 
expressed in camera with ultra-wide lenses. There are many analytical models for distortion modeling but when having a very large distortion value these models cannot clearly separate the influence of distortion from the influence of other sources of image coordinate errors. In this way, distortion influence corrupts other, interior and exterior orientation parameters of each image, and spoils the photogrammetric result. Therefore, this paper recommends first removing the influence of distortion on the images. For this purpose, the factoryrecommended distortion models are sufficient to use. Distortion removal in images is fully automated, off-line process, based on the same distortion model for all the images. Afterwards, precise distortion parameters should be determinate, simultaneously with interior and exterior orientation parameters. The procedure is performed by calculating phototriangulation with self-calibration based on appropriate, usually complex distortion models (Brown 1966, Torlegard 1981, TU Vienna). In this way, the accuracy and reliability of all unknowns of phototriangulation with self-calibration is increased 2 times, compared to one-step process (without initially removed distortion). It should be noted that numerous software solutions, developed specifically for creating 3D models, based on images captured with UAVs (Pix4D, Agisoft PhotoScan, Photomodeler), implement process for image distortion removal (idealized images) but only after an phototriangulation with self-calibration is completed. Because of previously mentioned facts, it is recommended to remove the distortion before using such software.

\section{REFERENCES}

Armstrong, M. N., 1996. Self-calibration from image sequences, Doctoral dissertation, University of Oxford, Oxford.

Azizi, N., 2003. Camera Self-Calibration, University of Toronto, Toronto.

Bendea, H., Boccardo, P., Dequal, S., Giulio Tonolo, F., Marenchino, D., \& Piras, M., 2008. Low cost UAV for postdisaster assessment, The International Archives of the Photogrammetry, Remote Sensing and Spatial Information Sciences, 37(Part B), 1373-1379.

Brown, D. 1966. Decentring Distortion of Lenses, Photo. Eng., $32,3,444-462$.

CCAA, 2015. Pravilnik o sustavima bespilotnih zrakoplova, Croatian Civil Aviation Agency, Narodne novine 49/2015.

Gajski, D. and Gašparović, M., 2015. Ispitivanje utjecaja distorzije objektiva nemjernih digitalnih kamera na točnost fotogrametrijske izmjere, Geodetski list 2015, 1, 27-40.

Kager, H., Rottensteiner, F., Kerschner, M., Stadler, P., 2002. ORPHEUS 3.2.1 User Manual, Institute of Photogrammetry and Remote Sensing, Vienna University of Technology, Vienna.

Karara, H.M., 1989. Non-Topographic Photogrammetry, 2nd Edition, ASPRS.

Kraus, K., 2006. Fotogrametrija 1. dio, Osnove i standardni procesi, Synopsis, Zagreb.

McGlone, J. C., 2013. Manual of Photogrammetry, 6th edition, American Society for Photogrammetry and Remote Sensing, Bethesda.
Perez, M., Aguera, F., Carvajal, F., 2013. Low cost surveying using an unmanned aerial vehicle, International Archives of the Photogrammetry, RS and Spatial information Sciences, Volume XL-1/W2, 2013 UAV-g2013, 4-6 September, Rostock, Germany.

Pollefeys, M., 1999. Self-calibration and metric 3D reconstruction from uncalibrated image sequences, Doctoral dissertation, ESAT-PSI, University of Leuven, Leuven.

Torlegard, K. (1981). Accuracy improvement in close range photogrammetry. Hochschule der Bundeswehr München.

Triggs, B., McLauchlan, P., Hartley, R., Fitzgibbon, A., 2000. Bundle Adjustment - A Modern Synthesis, Lecture Notes in Computer Science, 1883, 298-375.

Zalović, Đ., 2015. Sajam geodetske opreme - osobna komunikacija, Konferencija HKOIG, Opatija 2015.

URL 1: http://dronebuff.com/ 17.11.2015.

Revised April 2016 